Bull. Austral. Math. Soc.

46А $05,46 \mathrm{~A} 07$

VOL. 37 (1988) [383-388]

\title{
BARRELLED SPACES AND DENSE VECTOR SUBSPACES
}

\author{
W.J. Robertson, S.A. Saxon and A.P. Robertson
}

\begin{abstract}
This note presents a structure theorem for locally convex barrelled spaces. It is shown that, corresponding to any Hamel basis, there is a natural splitting of a barrelled space into a topological sum of two vector subspaces, one with its strongest locally convex topology. This yields a simple proof that a barrelled space has a dense infinite-codimensional vector subspace, provided that it does not have its strongest. locally convex topology. Some further results and examples discuss the size of the codimension of a dense vector subspace.
\end{abstract}

The notation and terminology are standard for the most part, as, for example, in [2] or [5]. We use $E$ for a locally convex Hausdorff space, $E^{\prime}$ for its (continuous) dual and $E^{\star}$ for its algebraic dual. The strongest (finest) locally convex topology is the Mackey topology $\tau\left(E, E^{\star}\right)$.

Any set of the form $\left\{\left(x_{i}, f_{i}\right): i \in A\right\} \subseteq E \times E^{\star}$ is a biorthogonal system if and only if $f_{i}\left(x_{j}\right)=1$ for $i=j$, and $f_{i}\left(x_{j}\right)=0$ for $i \neq j$.

THEOREM 1. Let $E$ be a Hausdorff barrelled space, the algebraic direct sum of the vector spaces $M$ and $N$. If there is a biorthogonal system $\left\{\left(x_{i}, f_{i}\right): i \in A\right\}$ such that $\left\{x_{i}: i \in A\right\}$ is a Hamel basis of $N$ and $\left\{f_{i}: i \in A\right\} \subseteq E^{\prime} \cap M^{\circ}$, then $N$ has its strongest locally convex topology and $E$ is the topological direct sum of $M$ and $N$.

Proof: Let $V$ be an absolutely convex absorbent set in $N$. If $i \in A$, there is some $\alpha_{i} \neq 0$ with $\alpha_{i} x_{i} \in V$. Put $y_{i}=\alpha_{i} x_{i}$ and $g_{i}=\alpha_{i}^{-1} f_{i}$; then $\left\{\left(y_{i}, g_{i}\right): i \in A\right\}$ is a biorthogonal system, $\left\{y_{i}: i \in A\right\}$ is a basis for $N$ and $\left\{g_{i}: i \in A\right\} \subseteq E^{\prime} \cap M^{\circ}$. Let $G$ be the polar of $\left\{y_{i}: i \in A\right\}$ in $\operatorname{span}\left\{g_{i}: i \in A\right\}$. Then $G^{\circ} \subseteq V+M$. For if $x \in G^{\circ}$, then $x=\sum_{i \in S} \lambda_{i} y_{i}+z$ for some finite subset $S$ of $A$ and $z \in M$, and $|g(x)| \leqslant 1$ for all $g \in G$. Take $g=\sum_{i \in S}\left(\overline{\operatorname{sgn}} \lambda_{i}\right) g_{i}$. Then $g \in G$ and $|g(x)|=\sum_{i \in S}\left|\lambda_{i}\right| \leqslant 1 ;$ thus $\sum_{i \in S} \lambda_{i} y_{i} \in V$ since $V$ is absolutely convex.

Also $G^{\circ}$ is absorbent since $M \subseteq G^{\circ}$ and each $y_{i} \in G^{\circ}$. Thus $G^{\circ}$ is a barrel since each $g_{i}$ is continuous, and so is a neighbourhood of the origin in $E$. Hence $V$ is a

Received 28 July, 1987

The work for this paper was begun when A.P. and W.J. Robertson were visiting the University of Florida; they would like to thank the University for its hospitality.

Copyright Clearance Centre, Inc. Serial-fee code: 0004-9729/88 \$A2.00+0.00. 
neighbourhood of the origin in $N, N$ has its strongest locally convex topology, and the projector of $E$ onto $N$ (along $M$ ) is continuous.

We note incidentally that the existence of such a biorthogonal system is also necessary for the conclusion of the theorem.

When $E$ is a locally convex space with Hamel basis $\left\{x_{i}: i \in B\right\}$, the corresponding biorthogonal system $\left\{\left(x_{i}, f_{i}\right): i \in B\right\}$ produces a natural splitting of $E$, determined by whether a linear form $f_{i}$ is continuous or discontinuous. Let

$$
\begin{aligned}
C=\left\{i: f_{i} \in E^{\prime}\right\}, & D=\left\{i: f_{i} \notin E^{\prime}\right\}, \\
E_{C}=\operatorname{span}\left\{x_{i}: i \in C^{\prime}\right\}, & E_{D}=\operatorname{span}\left\{x_{i}: i \in D\right\} .
\end{aligned}
$$

Then Theorem 1 has the following immediate consequence.

Theorem 2 (SPlitTing THEOREM). Let $E$ be a Hausdorff barrelled space and $\left\{x_{i}: i \in B\right\}$ a Hamel basis of $E$. Then, with the notation above, $E$ is the topological direct sum of $E_{C}$ and $E_{D}$, and $E_{C}$ has its strongest locally convex topology.

From Theorem 1 we also obtain:

COROLlARY. (Saxon-Levin $[8$, p.92]) Let $M$ be a closed vector subspace of counta.ble codimension in a Hausdorff barrelled space $E$. Let $N$ be any algebraic complement. of $M$ in $E$. Then $N$ is a topological complement of $M$ and has its strongest locally convex topology.

Proof: Given any basis $\left\{y_{1}, y_{2}, \ldots\right\}$ of $N$ we construct inductively a biorthogonal system satisfying the conditions of Theorem 1 . At each stage, $x_{n} \in \operatorname{span}\left\{y_{1}, \ldots, y_{n}\right\}$; the span of $M$ and the finite set $\left\{x_{i}: i<n\right\}$ is closed, and the Hahn-Banach theorem ensures the existence of a suitable $f_{n}$.

THEOREM 3. Let $E$ be a Hausdorff barrelled space and suppose that every vector subspace of $E$ has finite codimension in its closure. Then $E$ has its strongest locally convex topology.

Proof: Choose a Hamel basis $\left\{x_{;}: i \in B\right\}$ for $E$. With the notation of Theorem 2, $E$ is the topological sum $E_{C} \oplus E_{D}$, where $E_{C}$ has its strongest locally convex topology. Suppose that $D$ contains a countably infinite set $I$. Let $M=\operatorname{span}\left\{x_{i}: i \in\right.$ $B \backslash I\}$. By hypothesis, $\bar{M} \subseteq M+F$ where $F=\operatorname{span}\left\{x_{i}: i \in S\right\}$ for some finite subset. $S$ of $I$, and so $M+F=\bar{M}+F$ and is closed. Let $N=\operatorname{span}\left\{x_{i}: i \in I \backslash S\right\}$. Then by the Saxon-Levin Corollary, $E=(M+F) \oplus N$ and $N$ bas its strongest locally convex topology. Hence for each $i \in I \backslash S, f_{i}$ is continuous, which contradicts $I \subseteq D$. Therefore $D$ is finite and so $E_{D}$ is finite-dimensional; since, $E=E_{C} \oplus E_{D}, E$ has its strongest locally convex topology (and in fact $D$ is empty). 
CoRollary 1. If $E$ is Hausdorff and barrelled with $E^{\prime} \neq E^{\star}$ then there exists a dense $\aleph_{0}$-codimensional vector subspace $L$ of $E$; also there exists an $\aleph_{0}$-dimensional vector subspace $N$ of $E^{\star}$ with $N \cap E^{\prime}=\{0\}$ such that $L=N^{\circ}$.

Proof: Since $E^{\prime} \neq E^{\star}$ the theorem shows that there exists a vector subspace $M$ of $E$ with infinite codimension in $\bar{M}$. Let $K$ be an algebraic complement of $\bar{M}$ in $E$. Then $M+K$ is dense in $E$ and of infinite codimension. Let $L$ be a vector subspace containing $M+K$ and of codimension $\aleph_{0}$ in $E$.

Let $\left\{x_{i}: i \in I\right\}$ be a basis for an algebraic complement of $L$ in $E$, and $\left\{\left(x_{i}, f_{i}\right): i \in\right.$ I) be a biorthogonal system such that $f_{i}$ is zero on $L$ for all $i \in I$. Let $N=$ $\operatorname{span}\left\{f_{i}: i \in I\right\}$. Then $N \cap E^{\prime}=\{0\}$ since $L$ is dense, and clearly $L=N^{\circ}$.

We note that this Corollary shows that when $E$ has the Mackey topology $\tau\left(E, E^{\prime}+N\right)$ then $E$ is not barrelled. (For if $f$ is the pointwise limit of the lunconditional] series $\sum_{i \in I} f_{i}$, then $f$ is zero on the dense vector subspace $N^{\circ}$ and so is not in $E^{\prime}+N$; this is also a consequence of Theorem 4 of [7].) This provides an alternative proof that any barrelled topology on a space $E$ with $E^{\prime} \neq E^{\star}$ has a non-barrelled countable enlargement [6, Theorem 3].

CoROllary 2. If $E$ is a locally convex Hausdorff space and its associated barrelled topology [3] is not its strongest locally convex topology, then there exists a dense infinitecodimensional vector subspace of $E$.

(For by Corollary 1 , there is an infinite-codimensional vector subspace $L$, dense when $E$ has its associated barrelled topology, and therefore dense in the original topology on $E$.)

When $E$ is any locally convex Hausdorff space, not necessarily barrelled, with $E^{\prime} \neq E^{\star}$, then of course dense vector subspaces exist; it is easy to see that a dense vector subspace of finite codimension $n$ exists if and only if the codimension of $E^{\prime}$ in $E^{\star}$ is at least $n$. Some simple considerations limit the size of the codimension of a dense vector subspace in a similar way for the infinite case.

THEOREM 4. Let $E$ be a locally convex space with a dense $\aleph$-codimensional vector subspace, where $\mathcal{N}$ is any infinite cardinal. Then the codimension of $E^{\prime}$ in $E^{*}$ is at leasi $2^{\mathrm{N}}$.

Proof: Let $L$ be dense in $E$ with $K$-dimensional algebraic complement $M$. Then the vector subspace $L^{\circ}$ of $E^{\star}$ has dimension equal to that of $M^{*}$, which is $2^{\aleph}$. Since $L$ is dense, $L^{\circ} \cap E^{\prime}=\{0\}$, and so $\operatorname{codim} E^{\prime} \geqslant 2^{\mathrm{K}}$.

C'OROLlary 1. If $E^{\prime}$ has countable codimension in $E^{*}$, then $E$ has no dense vector subspace of infinite codimension. 
(Therefore in this case the intersection of a sequence of dense hyperplanes, the nullspace of a linearly independent sequence of linear forms, cannot be dense.)

CoRollaRY 2. If $E^{\prime}$ has codimension $2^{\aleph_{0}}$ in $E^{\star}$, then $E$ has no dense vector subspace of codimension $2^{\aleph_{0}}$. Thus, with the continuum hypothesis, in this case $E$ can have no dense vector subspace of uncountable codimension.

The theorem, with Corollary 2 of Theorem 3 , gives the following result.

C'OROLLARY 3. If the associated barrelled topology of $E$ is not $\tau\left(E, E^{\star}\right)$, then $\operatorname{codim} E^{\prime} \geqslant 2^{\aleph_{0}}$.

In $[\boldsymbol{\beta}]$, a theorem on completeness is used to deduce that the dual of a barrelled space is either $E^{\star}$ or has uncountable codimension in $E^{\star}$ [6, Theorem 2]. Here this result follows from Theorem 3, Corollary 1 and Theorem 4, Corollary 1.

Example. The following example, mentioned by Köthe [4, Section 22.5(5)] is cited by Eberhardt and Roelcke [1, 1.3] and also by Tsirulnikov [0, I, Note 2], who each demonstrate some of the points we raise here. However, we make this treatment selfcontained. First, we use it to show that the condition of Corollary 2 of Theorem 3, that the associated barrelled topology is not $\tau\left(E, E^{\star}\right)$, is not a necessary one for the existence of an infinite-codimensional dense vector subspace of $E$. In fact, there may even be a dense vector subspace of codimension equal to the dimension of $E$ when the associated barrelled topology is $\tau\left(E, E^{\star}\right)$.

(a). Let $I$ be an index set of uncountable cardinality $\aleph$, and let $E=\phi(I)$, the algebraic direct sum of $\aleph$ copies of the real numbers. Then $E^{\star}=\omega(I)=\mathrm{R}^{I}$. In $E^{\star}$, consider the vector subspace consisting of those functions with at most a countable number of non-zero coordinates. This is $\sigma\left(E^{\star}, E\right)$-dense in $E^{\star}$; call it $E^{\prime}$. For every countable subset $J$ of $I$, and for every absolutely convex absorbent subset $V$ of $\phi(J)$, let $U=V+\phi(I \backslash J)$, and give $E$ the topology with all such sets $U$ as a base of neiglıbourhoods of the origin.

(b). If $f$ is a linear form continuous for this topology, then $f$ is bounded on some $U$; hence $f$ has zero coordinates in the corresponding $I \backslash J$ and so $f \in E^{\prime}$. Conversely, if $f \in E^{\prime}$, there is a corresponding countable set $J$; let $U=\{x:|f(x)| \leqslant 1\}$. Then $U$ is of the form $V+\phi(I \backslash J)$ as above, and $f$ is bounded on $U$. Hence $E^{\prime}$ is the dual of $E$ with this topology.

(c). Now in $E$, take the Hamel basis $\left\{e_{i}: i \in I\right\}$ of coordinate vectors and the corresponding biorthogonal linear forms $f_{i}$. Since each $f_{i}$ has only one non-zero coordinate, $f_{i} \in E^{\prime}$ and so $f_{i}$ is continuous in the associated barrelled topology. Hence, by Theorem 2 applied to that topology, with the notation there, $E=E_{C}$ and $E$ has its strongest. locally convex topology. 
(d). Write $I=\bigcup\left\{I_{\gamma}: \gamma \in \Gamma\right\}$ where the $I_{\gamma}$ are disjoint, each has cardinality $\aleph$ and $\Gamma$ has cardinality $\aleph$. Let $h_{\gamma}(x)=\sum\left\{f_{i}(x): i \in I_{\gamma}\right\}$. Since the restriction of $h_{\gamma}$ to $\phi\left(I_{\gamma}\right)$ has an uncountable number of non-zero coordinates, it has no extension to $E$ which belongs to $E^{\prime}$; hence the restriction of $h_{\gamma}$ to $\phi\left(I_{\gamma}\right)$ is not continuous. Thus $h_{\gamma}^{-1}(0) \cap \phi\left(I_{\gamma}\right)$ is a dense hyperplane in $\phi\left(I_{\gamma}\right)$. Let $M=\bigcap\left\{h_{\gamma}^{-1}(0): \gamma \in I^{\prime}\right\}$. Then $h_{\gamma}^{-1}(0) \cap \phi\left(I_{\gamma}\right) \subseteq M$ and so $\phi\left(I_{\gamma}\right) \subseteq \bar{M}$ for all $\gamma$; hence $M$ is dense, and codim $M=\aleph$ (one dimension from each $\phi\left(I_{\gamma}\right)$; in fact we may choose any $i(\gamma)$ from each $I_{\gamma}$ and then $\operatorname{span}\left\{e_{i}: \gamma \in \Gamma\right\}$ is an algebraic complement of $M$ ).

It is attractive to conjecture that a locally convex space $E$, with the property that every $\aleph_{0}$-dimensional vector subspace is isomorphic to $\phi$ (that is, has its strongest locally convex topology) and has a topological complement,.must have topology $\tau\left(E, E^{*}\right)$. This example dispels that hope, even when the topology of $E$ is fine enough for the associated barrelled topology to be $\tau\left(E, E^{\star}\right)$, as we now show.

(e). Let $J \subseteq I$ be countable. Then clearly $\phi(J)$ is isomorphic to $\phi$, from the construction in (a) of the topology of $E$, and $E=\phi(J) \oplus \phi(I \backslash J)$ (algebraically and topologically). Suppose that $N$ is any $\aleph_{0}$-dimensional vector subspace of $E$. Then each element in a basis for $N$ is linearly dependent on only a finite number of coordinate vectors; so there is some countable $J$ such that $N \subseteq \phi(J)$. Let $M$ be an algebraic complement of $N$ in $\phi(J)$. Since $\phi(J)$ has its strongest locally convex topology, $M$ is also a topological complement. Hence $E=(N \oplus M) \oplus \phi(I \backslash J)=N \oplus(M \oplus \phi(I \backslash J))$.

(f). On the other hand, we may ask whether the conjecture is correct for barrelled spaces. However, in [1] Eberhardt and Roelcke define the class of GM-spaces, those $E$ for which the closed graph theorem holds for any linear mapping of $E$ into any metrisable locally convex space. Such spaces are therefore barrelled. In [1], it is shown that every $\aleph_{0}$-dimensional vector subspace of a GM-space is isomorphic to $\phi$ and has a topological complement (1.5), and that GM-spaces exist which do not have the strongest locally convex topology (3.5). In fact, Theorem 7 of $[\boldsymbol{\beta}]$ shows that, for a subclass of GM-spaces, there even exist dense vector sulspaces of codimension $2^{\mathbb{N}_{0}}$.

\section{REFERENCES}

[1] V. Eberhardt und W. Roelcke, 'Über einen Graphensatz für Abbildungen mit metrisierbarem Zeilraum', Manuscripta Math 13 (1974), 53-68.

[2] J. Horváth, Topological Vector Spaces and Distributions, 1 (Addison-Wesley, 1966).

[3] Y. Komura, 'On linear topological spaces', Kumamoto J. Sci. Ser. A 5 (1962), 148-157.

[4] G. Köthe, Topological Vector Spaces 1 (Springer-Verlag, 1969).

[5] A.P. Robertson and W.J. Robertson, Topological Vector Spaces (Cambridge University Press, 1964).

[6] W.J. Robertson, I. Tweddle and F.E. Yeomans, 'On the stability of barrelled topologies III', Bull. Austral. Math. Soc. 22 (1980), 99-112. 
[7] W.J. Robertson and F.E. Yeomans, 'On the stability of barrelled topologies I', Bull. Austral. Math. Soc. 20 (1979), 385-395.

[8] S. Saxon and M. Levin, 'Every countable-codimensional subspace of a barrelled space is barrelled', Proc. Amer. Math. Soc. 29 (1971), 91-96.

[ө] B. Tsirulnikov, 'On conservation of barrelledness properties in locally convex spaces', Bull. Soc. Roy. Sci. Liège 49 (1980), 5-25.

Dr. W.J. Robertson

Department of Mathematics

University of Western Australia

Nedlands, Western Australia 6009

Professor A.P. Robertson

School of Mathematical and Physical Sciences

Murdoch University

Murdoch, Western Australia 6150

Australia

\author{
Di. S.A. Saxon \\ Department of Mathematics \\ University of Florida \\ Gainesville, Florida 32611 \\ U.S.A.
}

